\title{
Unusual Clinical Tumor Development in Axilla
}

\section{Strunová M. ${ }^{1}$, Pavlišta D. ${ }^{1}$, Janoušek M. ${ }^{1}$, Haškovcová J.2, Ulrych V. ${ }^{3}$, Kobilková J. ${ }^{1}$}

${ }^{1}$ Oncogynecological Centre, Department of Obstetrics and Gynaecology, First Faculty of Medicine, Charles University in Prague and General University Hospital in Prague, Prague, Czech Republic;

2Institute of Pathology, First Faculty of Medicine, Charles University in Prague and General University Hospital in Prague, Prague, Czech Republic; 3 Multiscan, Oncological Clinic Pardubice, Pardubice, Czech Republic

Received May 30, 2013; Accepted August 14, 2013.

Key words: Axilla - Sarcoma - Neurogenic sarcoma

Abstract: This work describes clinical development of a sarcoma in the left axilla of a 36-year-old woman. The macroscopic picture changed from the initial inflammatory reddening to globular resistance of $2.5 \mathrm{~cm}$, suggestive of an enlarged lymph node. Mammography did not reveal any associated breast disease. Colliquation found on the ultrasound images led to a biopsy, the result of which indicated only an inflammation, without any malignancy. Rapid growth of the axillar tumor to $10 \mathrm{~cm}$ in size within 8 weeks prompted surgery allowing proper diagnosis of a small mature-to-immature sarcoma. Special examinations performed by a histopathologist (at the Institute for Histopathology) could not establish the precise histogenesis, i.e. the tissue origin. Therefore it was necessary to remove any clinically obscuring tumor for the final proper histological diagnosis and adequate treatment of the patient.

Mailing Address: Marie Strunová, MD., Oncogynecological Centre, Department of Obstetrics and Gynaecology, First Faculty of Medicine, Charles University in Prague and General University Hospital in Prague, Apolinářská 18, 12800 Prague 2, Czech Republic; Mobile Phone: +420 774886 511; Fax: +420 224967 452; e-mail: marie.strunova@vfn.cz 


\section{Introduction}

In the personal anamnesis of our 36-year-old patient (G2/P2) no relation to the present ailment was found. The gynecological anamnesis showed two spontaneous child births, after the second birth a conization was performed, which had a histological finding of CIN 2. The patient used hormonal contraceptives, which was changed after the conization to IUD - Mirena. The patient's weight was $65 \mathrm{~kg}$, and her height was $165 \mathrm{~cm}$.

At a regular gynecological check-up the patient indicated an inflammatory lesion in her left armpit, with a reddish area reminiscent of folliculitis. The patient was given local treatment, and subsequently the inflammation subsided. 3 weeks later the patient returned having found an axillar resistance which was movable with restrictions. The skin over the formation was without any pathological findings.

On 23 April 2012 there a mammography was performed to establish any correlation with the breast area. The mammographic type was glandular; an ultrasonic check discovered a small cyst to the left in the top outside quadrant, while in the right breast two fibromas about $4 \mathrm{~mm}$ in size were found in the top outside quadrant behind the nipple areola, and in the left axilla there was a globular hypoechoic node with slight vascularization in the periphery, $28 \mathrm{~mm}$ in size, of an infiltrated character. After consulting the mammography center, a biopsy using an ultrasound was undertaken. Puncture samples from the resistance were processed into a block and sliced up in their entirety. Microscopic image showed ligament and adipose tissue with vessels and nerves and sparse uncharacteristic globo-cellular cellularization. No structures of lymph nodes were present, and the samples did not contain any tumor growth. The patient came for a check-up two weeks later. Owing to the negative histological result, and based on the confirmed chronic nodes in the right axilla, we performed only a supplementary examination, resulting in the decision to remove the resistance in the left axilla. Laboratory examination and blood tests showed neutrophils 76.3/standard 50-75.0, monocytes 9.5/standard 3-8, lymphocytes 0.58/standard 1.00-4.8. Serum tumor markers Ca 19-9 53/ standard 37.0, Ca 15-3, CEA, Ca 125, Ca 72-4 negative.

The patient was recommended to undergo surgery, i.e. extirpation of the formation; however she left for a planned holiday and returned 8 weeks later. By then the resistance had grown to $10-12 \mathrm{~cm}$. The patient explained it by excessive physical activity. The tumor extirpation was performed on 16 July 2012. The axilla tumor was about 9-10 cm, semi-fixed, reaching the chest wall. The area around the tumor was more perfused; at the bottom it was partly fixed by $2 \times 2 \mathrm{~cm}$. During surgery the housing was slightly damaged at the site of fixation to the chest wall. In the center the tumor was necrotic and was removed with macroscopically healthy tissue.

Histology from 16 July 2012 revealed a bulky, partly lipid particle $90 \times 60 \times 20-30$ mm, a whitish irregular deposit on the section, probably tumorous, up to $40 \mathrm{~mm}$ in diameter. In the axilla excision there was a large tumor focus with the structure 

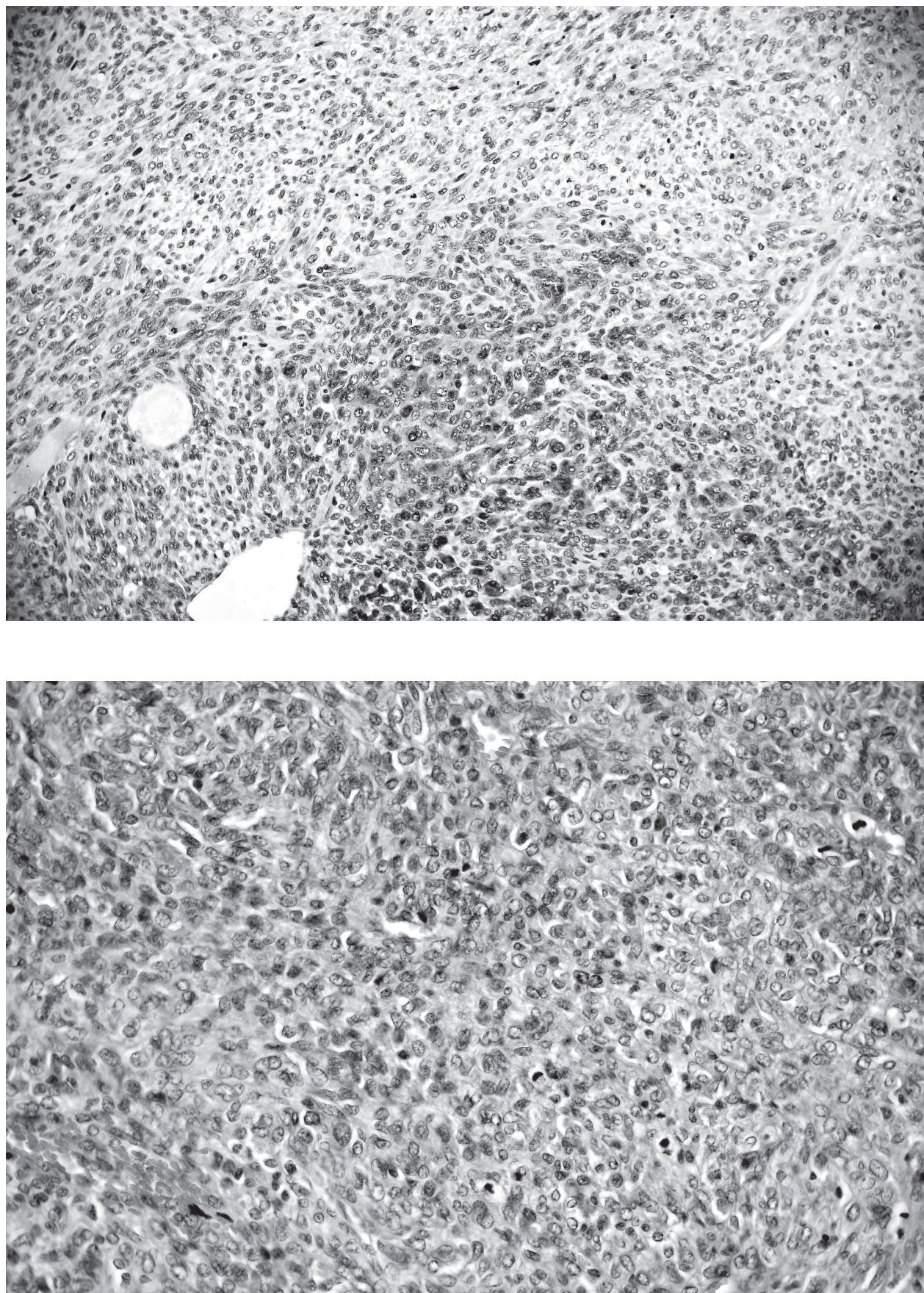

Figures 1 and 2 - The diagnosis of spindle cell sarcoma in left axilla (Institute of Pathology, First Faculty of Medicine, Charles University in Prague and General University Hospital in Prague Assoc. Prof.J. Haškovcová, MD., PhD.). 
of the spindle cell sarcoma, moderately to poorly differentiated. Its starting point was uncertain, and wasn't made absolutely clear even by an immune-histochemical examination, which had mainly negative results in reaction with CKAE, CAM5.2, EMA, CD34 and alpha-actin. S-100 protein was, however, focally positive in the nuclea and cytoplasm, which aroused suspicion of neurogenetic origin (Figure 1). The result of an additional immune-histochemical examination showed chromogranin, synaptophysin, Bc12 and calponin as negative. These examinations do not, however, compromise the original diagnostic conclusion that this was most probably a case of neurogenic sarcoma, even though this conclusion is not entirely final.

When healed, the patient was transferred to oncology for staging procedures - CT of the chest, belly and small pelvis. The pulmonary parenchyma showed no evidence of oncological changes; two tiny nodulations up to $3 \mathrm{~mm}$ in size were located on the left in the apex, subpleurally, while there were also two tiny nodulations on the right in the apex dorsally. The tiny and evidently hypodensic focus in S7 of the liver indicates the probability of a cyst. Other focal changes in the liver were not demonstrated, and the skeleton showed no destructive changes. The bile ducts, pancreas, kidney and adrenal glands did not demonstrate any tumorous changes, either. The uterus was not enlarged, and the IUD was in situ. The ultrasound with color mapping showed a slightly irregular inhomogeneous structure in the area of the surgical scar, along with lower hypogenity in soft axilla tissues without traces of pathological vascularisation. These are probably only postsurgical changes. Neither axilla shows any sign of lymphadenopathy. PET/CT established in the left axilla an infiltrate of $20 \mathrm{~mm}$ size with a raised accumulation of FDG, as well as several small nodules of up to $7 \mathrm{~mm}$ in size, also with raised accumulation of FDG. There is also a slight asymmetry in consumption of FDG in the breasts subareolarly, with the left side unaffected. Based on these findings a punctual biopsy was performed from the indicated points (infiltrate + nodules): no tumor pathology was found.

The next step was radiotherapy. The left axilla was treated with a linear accelerator from three shaped areas by $50 \mathrm{~Gy}$ with a subsequent boost. The patient tolerated the radiotherapy very well; towards the end the skin showed slight erythematous reaction in the axilla and the submammary groove. At the moment the patient is without any pathological findings. Length of follow up is 9 months.

\section{Discussion}

The axillar area is an anatomically complex structure containing lymphatic system, vascular and neural structures and adipose fibrous tissue; it is covered with skin with adnexa. The apparent inflammation was probably related to the tumor necrosis described by the surgeon at the axillar tumor surgery. The initial phase of sarcoma in the armpit appeared initially as an inflammatory process of the skin 
adnexa. It is probable that the puncture needle was inserted into the necrotic center of the tumor. However, it is interesting that the visible inflammatory skin image subsided after local therapy, while at a greater depth there was a palpable tumor $4 \mathrm{~cm}$ in size. Due to the postponement of surgery, the formation grew in size.

Tumor sarcomas are rare in the axillary region. Finding the resistance firstly indicates metastases of lymphatic nodes in the breast, hematological malignity, inflammatory lesion and differential-diagnostically also a nodular syndrome. This tumor was characterized by a rapid growth. The word sarcoma comes from the Greek sarx (meat), because most sarcomas have the appearance of meat. These tumors are usually very immature; in microscopic image they have the structure of mesenchymal tissue. The spindle cell sarcoma of our patient described by the histopathologist has elongated narrow cells with little cytoplasm. The cells have hyperchromatic cores; they often have fine needle aspirations which are in connection with other cells (Figure 2). Spindle cells are histologically organized in parallel into bundles (the fascicular arrangement). In histogenetics we can nowadays classify the cells of a spindle cell sarcoma (originally a purely descriptive term) by means of immune-histochemistry. Sarcomas are immature mesenchymal tumors which are grouped according to the maternal tissue from which they originate (fibroma, myxoma, lipoma from the peripheral nervous system, serous membranes together with further tumors of other categories). Therefore the sarcoma in the axillar area can originate from different tissues (Horton and Hill, 1979; Povýšil and Šteiner, 2011).

Vimentin is a good general marker for nonmuscle sarcomas in FNA (fine needle aspiration). All nonmuscles sarcomas are vimentin positive. However, vimentin immunoreactivity needs careful interpretation (Bibbo, 1997). With conventional light microscopy and clinical data we estimate that a definitive cytologic diagnosis of tumor type can be provided for $85-95 \%$ of FNA material. Leaving $10-15 \%$ of "difficult cases" (Bibbo, 1997). Soft tissue and bone tumors arise from derivates of embryonic mesoderm and are composed of heterologous cellular elements, neoplastic cells and entrapped cells of local host tissues at different stages of differentiation. This is the cell morphology; tissue patterns and overall composition of tumors are subject to modulation and can be influenced by local tissue conditions and anendless number of other factors (Hajdu, 1980). Those are the diagnostic difficulties, which were met also in our case.

Special examination methods were not able to determine the exact origin of the immature, rapidly-growing tumor; the immunohistochemical examination indicated the origin to be a neurogenic sarcoma. After consulting the surgeon we came to the conclusion that the original tissue of the sarcoma was most probably the axilla ligament where the tumor had a consistent base. We found only one report about sarcoma in this localization, but the histological type was different liposarcoma (Manabe et al., 2005; Davidescu et al., 2011; Campbell et al., 2012). 
We have not found any work on neurogenic sarcoma in the armpit in any of the available literature published in the last 10 years. In this regard, this case illustrates rare localization of neurogenic sarcoma.

\section{Conclusion}

This work presents the rare localization of a sarcoma which, in its initial stage, had the features of a banal inflammation. After the subsiding of the clinical inflammation the resistance in the armpit was enclosed and led us to consider its relation to the mammary gland, based on the image of an enlarged node. The biopsy from the resistance confirmed the inflammatory etiology. The final diagnosis was determined by surgical extirpation.

\section{References}

Bibbo, M. (1997) Comprehensive Cytopathology. W.B. Saunders, Philadelphia.

Campbell, G. S., Lawrence, T. J., Porter, S. E., Rezeanu, L. (2012) Primary dedifferentiated liposarcoma of the axilla arising in a mixed, well-differentiated and myxoid liposarcoma. J. Radiol. Case Rep. 6(1), 9-16.

Davidescu, M., Motas, N., Bluoss, C., Motas, C., Rus, O., Horvat, T. (2011) Neurogenic tumors of the posterior mediastinum. Chirurgia (Bucur.) 106(2), 199-203. (in Romanian)

Hajdu, S. I. (1980) The paradox of sarcoma. Acta Cytol. 4, 373-383.

Horton, J., Hill, J. (1979) Klinická Onkologie. Avicenum, Praha.

Manabe, H., Umemoto, T., Takagi, H., Matsuno, Y., Kato, T., Sekino, S., Sekido, Y. (2005) Primary neurogenous sarcoma of the lung; report of a case. Kyobu Geka 58(4), 337-340. (in Japanese)

Povýšil, C., Šteiner, I. (2011) Obecná Patologie. Galén, Praha. 